\title{
Can temporal repetitive transcranial magnetic stimulation be enhanced by targeting affective components of tinnitus with frontal rTMS? A randomized controlled pilot trial
}

\author{
Peter Michael Kreuzer ${ }^{1}{ }^{*}$, Michael Landgrebe ${ }^{1}$, Martin Schecklmann ${ }^{1}$, Timm B. Poeppl ${ }^{1}$, \\ Veronika Vielsmeier ${ }^{2}$, Goeran Hajak ${ }^{1,3}$, Tobias Kleinjung ${ }^{2,4}$ and Berthold Langguth ${ }^{1}$ \\ ${ }^{1}$ Department of Psychiatry and Psychotherapy, University of Regensburg, Regensburg, Germany \\ 2 Department of Otolaryngology, University of Regensburg, Regensburg, Germany \\ ${ }^{3}$ Department of Psychiatry, Psychosomatic Medicine and Psychotherapy, Bezirkskrankenhaus Bamberg, Bamberg, Germany \\ ${ }^{4}$ Department of Otolaryngology, University of Zurich, Zurich, Switzerland
}

Edited by:

Jos J. Eggermont, University of Calgary, Canada

Reviewed by:

Dirk De Ridder, University Hospital Antwerp, Belgium

Robert Folmer, Portland VA Medical Center, USA

\section{${ }^{*}$ Correspondence:}

Peter Michael Kreuzer, Department of Psychiatry and Psychotherapy,

University of Regensburg;

Universitaetsstr. 84, 93053

Regensburg, Germany.

e-mail:peter.kreuzer@medbo.de

\begin{abstract}
Objectives: Low-frequency repetitive transcranial magnetic stimulation (rTMS) of the temporal cortex has been investigated as a new treatment tool for chronic tinnitus during the last years and has shown moderate efficacy. However, there is growing evidence that tinnitus is not a pathology of a specific brain region, but rather the result of network dysfunction involving both auditory and non-auditory brain regions. In functional imaging studies the right dorsolateral prefrontal cortex has been identified as an important hub in tinnitus related networks and has been shown to particularly reflect the affective components of tinnitus. Based on these findings we aimed to investigate whether the effects of left low-frequency rTMS can be enhanced by antecedent right prefrontal low-frequency rTMS. Study Design: Fifty-six patients were randomized to receive either low-frequency left temporal rTMS or a combination of low-frequency right prefrontal followed by low-frequency left temporal rTMS. The change of the tinnitus questionnaire (TQ) score was the primary outcome, secondary outcome parameters included the Tinnitus Handicap Inventory, numeric rating scales, and the Beck Depression Inventory. The study is registered in clinicaltrials.gov (NCT01261949). Results: Directly after therapy there was a significant improvement of the TQ-score in both groups. Comparison of both groups revealed a trend toward more pronounced effects for the combined group (effect size: Cohen's $d=0.176$ ), but this effect did not reach significance. A persistent trend toward better efficacy was also observed in all other outcome criteria. Conclusion: Additional stimulation of the right prefrontal cortex seems to be a promising strategy for enhancing TMS effects over the temporal cortex. These results further support the involvement of the right DLPFC in the pathophysiology of tinnitus. The small effect size might be due to the study design comparing the protocol to an active control condition.
\end{abstract}

Keywords: chronic tinnitus, dorsolateral prefrontal cortex, limbic system, transcranial magnetic stimulation, rTMS, neuromodulation

\section{INTRODUCTION}

Subjective tinnitus is characterized by the perception of sound or noise in the absence of an objective physical sound source (Moller, 2003). There is convincing evidence from functional imaging (Crippa et al., 2010; Lanting et al., 2010) and neurophysiologic studies (Weisz et al., 2007a,b) that tinnitus is related to abnormal functioning of the central auditory system (Moller, 2003). Based on these findings repetitive transcranial magnetic stimulation (rTMS) of the temporal and temporoparietal cortex has been proposed as a potential treatment for chronic tinnitus (Eichhammer et al., 2003).

Transcranial magnetic stimulation (TMS) is a non-invasive tool for inducing electric currents in the brain (Hallett, 2000). Fast oscillating magnetic fields created by a strong electric current circulating within a coil, penetrate the skull and result in depolarization of superficial cortical neurons (Ridding and Rothwell,
2007). rTMS can induce alterations of neuronal activity that outlast the actual stimulation period for a considerable amount of time (Hallett, 2000). Therefore, this technique has gained increasing attention as a potential clinical tool for the treatment of different neuropsychiatric disorders. Although the direct effects of the magnetic field are limited to directly stimulated superficial brain areas (Siebner et al., 2003), indirect effects can also occur in functionally connected remote areas (Hallett, 2000; Siebner et al., 2000). Such remote stimulation effects have also been demonstrated in thalamic regions after temporal rTMS by using voxel-based morphometry (May et al., 2007).

Several clinical studies consistently showed a reduction of tinnitus severity after repeated $1 \mathrm{~Hz}$ rTMS applied to the temporal cortex, whereas sham treatment had no effect (Kleinjung et al., 2005; Plewnia et al., 2007; Rossi et al., 2007; Smith et al., 2007). However, treatment results are burdened by only moderate 
improvement and high inter-individual variability indicating the need for optimization strategies.

As hypothesized already more than 20 years ago (Jastreboff, 1990) and confirmed by recent neuroimaging findings, tinnitus is related to (i) abnormal activity in both auditory and non-auditory brain regions (Lanting et al., 2009; Leaver et al., 2011) and to (ii) abnormal functional connectivity between these regions (Schlee et al., 2008, 2009a,b; De Ridder et al., 2011). In these studies the right dorsolateral prefrontal cortex has been identified as an important hub (Schlee et al., 2008, 2009a,b; Vanneste et al., 2010a). It has been hypothesized that this area might especially be related to the affective components of tinnitus (Vanneste et al., 2010a; De Ridder et al., 2011; Langguth et al., 2011). It has even been speculated that based on the emotional relevance, involved limbic and paralimbic structures may effectively switch the perceived signal on and off (Rauschecker et al., 2010).

This is in line with electrophysiological studies that demonstrated the relevance of dysfunctional top-down inhibitory mechanisms originating in the prefrontal lobe for tinnitus generation (Norena et al., 1999). The critical relevance of the DLPFC for tinnitus annoyance has been affirmed by recent studies that demonstrated symptom reduction after bifrontal tDCS (Vanneste et al., 2010b; Frank et al., 2011). Furthermore, it has been shown that rTMS over the DLPFC is apt to modulate the activity in functionally connected central limbic pathways such as the anterior cingulated cortex (Paus et al., 2001). Modulation of neuronal activity in the anterior cingulate, parahippocampus, and auditory cortex has also been reported in tinnitus patients after transcranial direct current stimulation of the prefrontal cortex (Vanneste and De Ridder, 2011).

A further rationale for low-frequency stimulation of the right DLPFC derives from affective research. Frontal asymmetry is known to influence emotion regulation and the emotional reaction to sensory stimuli (Davidson, 1992; Schmidt and Hanslmayr, 2009). It has also been shown that low-frequency rTMS of the right DLPFC exerts antidepressant effects of similar magnitude like high frequency rTMS of the left DLPC, which is conventionally applied in depressive disorders (Schutter, 2010).

Based on these data and the right lateralized alterations of frontal cortex activity in tinnitus patients (Schlee et al., 2008) we hypothesized that low-frequency rTMS of the right DLPFC might enhance treatment effects of low-frequency rTMS in tinnitus patients and compared the combined prefrontal and temporal
rTMS therapy in tinnitus patients with the standard procedure of temporal rTMS.

\section{PATIENTS AND METHODS}

Fifty-six patients with chronic unilateral or bilateral tinnitus were enrolled in the study after having given written informed consent. The study has been registered with clinicaltrials.gov (http://clinicaltrials.gov/ct2/show/NCT01261949), was approved by the local ethics committee and performed according to the declarations of Helsinki. All patients suffered from disturbing tinnitus and had tried several standard treatment modalities such as cognitive behavioral therapy, hearing aids, white-noise generators, vasodilators, or antidepressants in the past. Normal middle ear status was demonstrated by tympanometry, stapedius reflex tests, and otoscopy. Patients with a history of seizures, a suspected diagnosis of organic brain damage, as well as patients with cardiac pacemakers, mobile metal implants, or implanted medication pumps were excluded. Sample characteristics are provided in Table 1. All data in the text and table is given as mean \pm SD. rTMS was applied with the use of a Medtronic system with a figure-8 coil (Cool B-65 Butterfly; Medtronic, Minneapolis, MN, USA). Patients were enrolled in the study on a Monday and received stimulation on 10 subsequent working days. Patients were randomly assigned to one of two treatment protocols. One protocol (standard protocol) consisted of 2000 stimuli at a frequency of $1 \mathrm{~Hz}$ and an intensity of $110 \%$ resting motor threshold (RMT) over the left auditory cortex. In the second treatment protocol (combined protocol), low-frequency stimulation (1000 stimuli, $1 \mathrm{~Hz}, 110 \%$ motor threshold) applied to the right DLPFC preceded left temporal stimulation (1000 Stimuli, $1 \mathrm{~Hz}, 110 \%$ RMT). Thus, the total number of applied stimuli per session was identical for both groups. Stimulation was administered over the right DLPC and the left temporal cortex regardless of handedness or tinnitus laterality (Kleinjung et al., 2007b, 2008). The handle of the coil was pointing upward. Thus, the induced current in the brain was directed approximately perpendicular to the location of the superior temporal gyrus. During treatment the coil was held with a mechanical arm. In the combined stimulation group, the TMS coil was localized over the right DLPFC according to a standard algorithm by moving the coil from the optimal position for stimulation of the left abductor minimi $6 \mathrm{~cm}$ in the anterior direction and transferring this spot to the contralateral hemisphere in respect of the distance to the sagittal axis of the skull (George

Table 1 | Clinical and demographic characteristics (mean \pm SD).

\begin{tabular}{|c|c|c|c|c|c|}
\hline & $1 \mathrm{~Hz}$ & $1 / 1 \mathrm{~Hz}$ & $T / \chi^{2}$ & df & $\mathbf{p}$ \\
\hline Age & $46.5 \pm 14.9$ & $51.1 \pm 13.9$ & 1.196 & 54 & 0.237 \\
\hline Preceding treatments & $2.5 \pm 0.95$ & $2.9 \pm 0.56$ & 2.063 & 53 & 0.044 \\
\hline Gender (male/female) & $23 / 7$ & $19 / 7$ & 0.096 & 1 & 0.757 \\
\hline Tinnitus laterality (right/left/both) & $2 / 3 / 22$ & $1 / 7 / 14$ & 3,235 & 2 & 0.198 \\
\hline TQ total score at baseline & $39.5 \pm 17.7$ & $35.9 \pm 17.1$ & -0.774 & 54 & 0.442 \\
\hline Audiogram (average from $125 \mathrm{~Hz}$ to $8 \mathrm{kHz}$ of both ears) & $20.8 \pm 14.0$ & $25.5 \pm 17.8$ & 0.650 & 54 & 0.519 \\
\hline
\end{tabular}


et al., 1995). The RMT was determined over the left motor cortex for the right abductor digiti minimi and defined as the lowest intensity at which at least four of eight consecutive MEPs were $50 \mathrm{mV}$ in amplitude while the muscle being investigated was at rest. Tinnitus severity was assessed before treatment (baseline), at the end of treatment (week 2), and during a certain follow-up period after rTMS treatment (week 4 and week 12). Tinnitus assessments included the German versions of the tinnitus questionnaire (TQ; Goebel and Hiller, 1994), the Tinnitus Handicap Inventory (Kleinjung et al., 2007a), the Beck Depression Inventory (Beck and Steer, 1984), several tinnitus numeric rating scales (loudness, discomfort, annoyance, distractibility, unpleasantness; Landgrebe et al., 2010), and a quality of life scale (WHOQOL-BREF; Murphy et al., 2000).

\section{STATISTICAL ANALYSIS}

The data analysis was based on data of the Tinnitus Research Initiative Database. Data management was conducted according to the Data Handling Plan (TRI-DHP V07, May 9th, 2011). Data analysis was conducted according to the Standard Operating Procedure (TRI-SA V01, May 9th, 2011), thereby following a study-specific Statistical Analysis Plan (SAP-003, May 18th, 2011) that was written according to the SAP template (TRI-SAP V01, May 12th, 2011). All documents are to be found under http://database.tinnitusresearch.org/.

The statistical analysis was performed on an intention-to-treat basis including all patients who participated in at least one measurement time point using a last observation carried forward or backward approach. Primary outcome was the change in TQ scores from baseline to week 2. For this purpose, we conducted an analysis of variance (ANOVA) with the within-subjects factor time (baseline vs. week 2) and the between-subjects factor group (combined vs. temporal group). Secondary outcome measures and exploratory analyses included chi-square tests for the variables group and treatment response which was defined as amelioration of at least 5 points in the TQ. We pooled the group of responders and non-responders of both treatments and compared them in regard to demographic and clinical characteristics with chi-square and $t$-tests. Furthermore, we compared baseline corrected TQ scores (week 2 minus baseline) between the treatment groups with Student's $t$-tests. In addition, we again conducted an ANOVA with the factor group (between-subjects factor) and time (within-subjects factor), this time including five measurement time points (screening, baseline, week 2, week 4, and week 12). This ANOVA was also computed for all other secondary outcome parameters (i.e., THI, BDI, and WHOQoLBREF). Furthermore, we compared baseline corrected TQ scores (week 2 minus baseline; week 4 minus baseline) between the treatment groups. The statistical threshold for alpha error was set at 0.05 . The analysis of secondary outcome parameters followed an exploratory approach, without corrections for multiple comparisons.

\section{RESULTS}

Both stimulation protocols were well tolerated, and all patients except one completed the treatment. This patient (combined stimulation group) refused further stimulation after day 3 because she feared a possible deterioration of the symptoms. A total of seven patients (including the one mentioned before) did not complete the course of the study (not shown up for follow-up-visit without giving further explanation). Three of them were treated in the combined stimulation group, four in the conventional group. Transient mild to moderate headache and feelings of twitching muscles at the stimulation site were reported as side effects. Serious adverse or side effects were not observed.

Primary outcome analysis indicated a significant change over time for both groups as indicated by a significant main effect of time $(F=6.1 ; \mathrm{df}=1.54 ; p=0.017)$, but no group differences (main effect of group: $F=0.8$; $\mathrm{df}=1.54 ; p=0.375$; interaction effect time by group $F=0.434 ; \mathrm{df}=1.54 ; p=0.513$ ).

Response rate was comparable between groups (combined: $40 \%$; temporal: $\left.37 \% ; \chi^{2}=0.1 ; \mathrm{df}=1 ; p=0.800\right)$. Effect sizes were near zero for the non-responder groups (combined: $d=0.085$; temporal: $d=0.104$ ) and medium to high for the responder groups (combined: $d=0.700$; temporal: $d=0.454$ ). Contrasts between these groups indicated no significant differences for age, gender, tinnitus laterality, duration, and hearing loss. We only found an effect for the change in TQ and THI from screening to baseline for the non-responder group in contrast to the responder group of the combined treatment (TQ: $T=2.156$; $\mathrm{df}=24 ; p=0.041$; THI: $T=3.675 ; \mathrm{df}=24 ; p=0.001)$, i.e., there was a reduction of questionnaire scores from screening to baseline for the non-responder (TQ: $-5.2 \pm 11.7$; THI: $-5.6 \pm 7.7$ ) and an increase for the responder group (TQ: $3.5 \pm 6.1$; THI: $5.8 \pm 8.0)$

Comparable to the primary outcome analysis, ANOVAs with five time points for TQ, THI, and BDI indicated significant main effects of time (all Fs $>2.3$; df $=4.212$; all $p s<0.065$ ) and neither significant effects of group (all $F s<2.6$; $\mathrm{df}=1.53$; all $p s>0.115$ ) nor time by group (all $F \mathrm{~s}<0.9$; df $=4.216$; all $p s>0.462$ ). Post hoc tests indicated an amelioration of symptoms after beginning of treatment and a return to baseline levels during the last followup (see Figure 1), i.e., tinnitus scores were significantly bettered for week 2 and week 4 in contrast to screening, baseline and follow-up.

Baseline corrected group contrasts (week 2 minus baseline; week 4 minus baseline) indicated no significant differences for week 2 (all $p$ s $>0.265$ ) and week 4 (all $p$ s $>0.088$ ) for TQ, THI, and BDI. Range of effect sizes were between 0.168 and 0.461 ( 0.176 for primary outcome analysis) indicating more pronounced improvement for the combined group in contrast to the temporal group for all variables.

\section{DISCUSSION}

The main finding of this trial is that additional low-frequency stimulation of the right DLPFC failed to significantly improve the effects of low-frequency temporal stimulation in the treatment of tinnitus. However, on a descriptive level the combined treatment protocol yielded better results in all assessment instruments that have been applied. Primary outcome analysis showed an effect size of 0.176 (group contrast in week 2) indicating a small effect according to Cohen (1988). Thus, the effect size was 


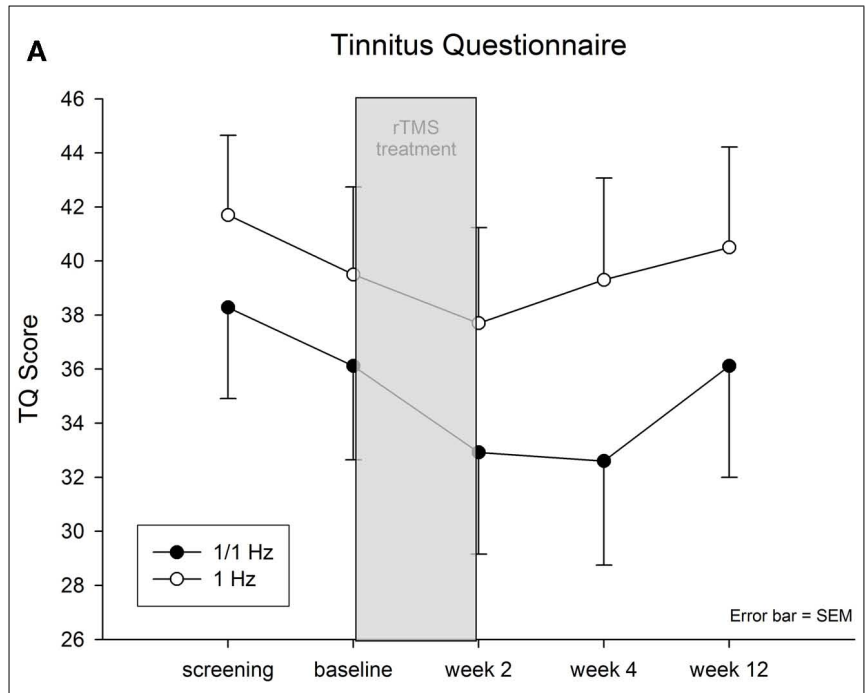

B

Tinnitus Handicap Inventory
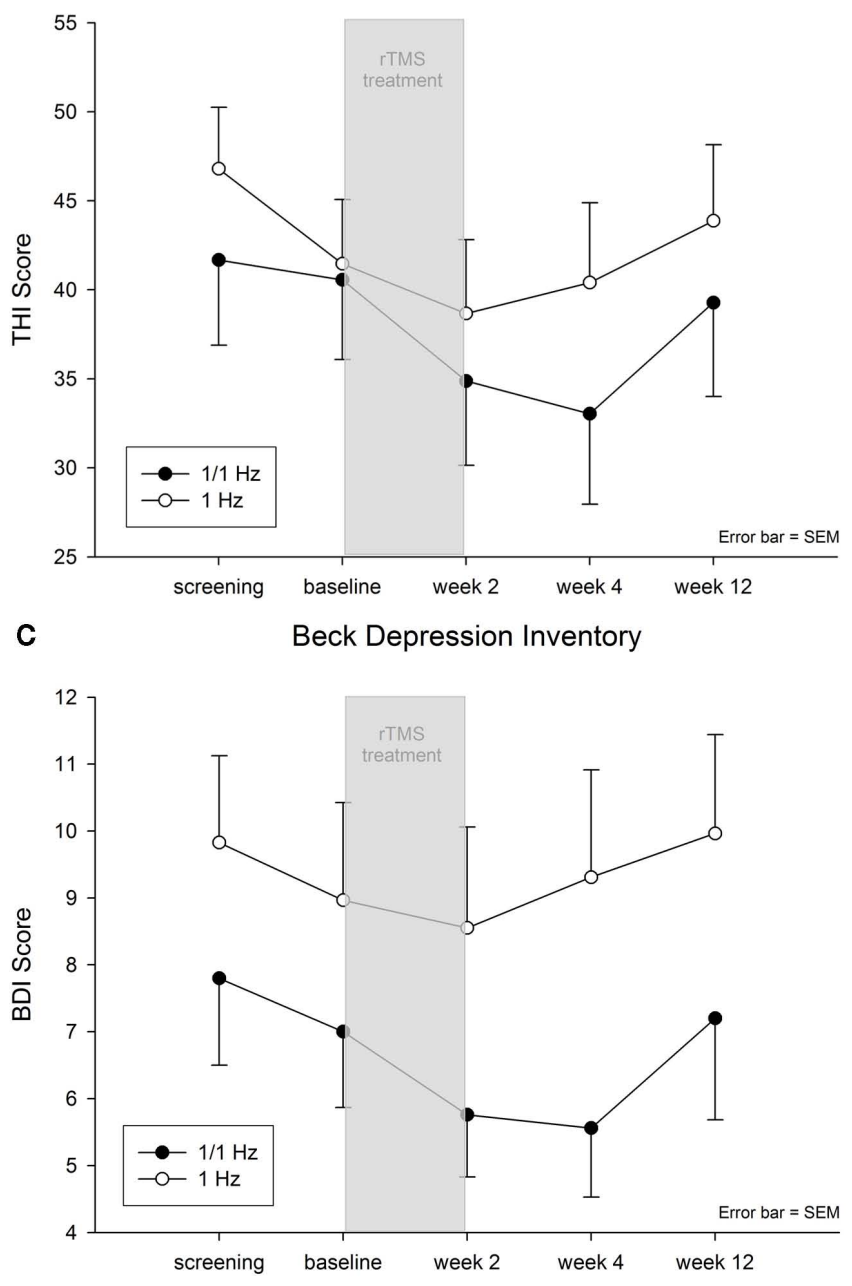

FIGURE 1 | (A) Tinnitus Questionnaire Score (TQ; mean \pm SEM), (B) Tinnitus Handicap Inventory (THI; mean \pm SEM), and (C) Beck Depression Inventory (BDI; mean \pm SEM). smaller than expected resulting in limited power and the failure to demonstrate significant effects. In this context it should be noted that significant improvement after rTMS was observed in both stimulation groups. The effect size for responders from temporal stimulation was medium $(d=0.45)$ for the responders from the combined stimulation protocol high $(d=0.70)$ according to Cohen (1988). Interestingly, the responder group differed significantly from non-responders in the change of THI and TQ scores from screening to baseline (non-responders: mean effect $-4 \mathrm{TQ} / 7 \mathrm{THI}$ points; responders: mean effect $+2 \mathrm{TQ} / \mathrm{THI}$ points). This might be interpreted as a hint for the induction of homeostatic effects by TMS (Siebner et al., 2004). Further analysis did not reveal any differences between responders and non-responders in respect to gender, age, tinnitus duration, laterality, number of previous treatments, hearing loss, numeric rating scales, and values (screening/baseline) for THI, $\mathrm{BDI}$, and TQ.

A further important finding was the decrease of mean baseline values for TQ, THI (for the $1 / 1-\mathrm{Hz}$ group), and BDI from screening to baseline. This effect was similar in both stimulation groups and may have several reasons. First screening scores are based on completion of the TQ at home before the first consultation in our tinnitus clinic. The examination and consultation in the tinnitus clinic, which also involves counseling, may have resulted in the reduction of the tinnitus scores. Alternatively the improvement can be interpreted as an anticipation effect. Similar effects were observed in patients enrolled in waiting list control groups (Hesser et al., 2011).

Facing the fact that the combined stimulation group has been compared to an actively treated control group [that has undergone an already established standard treatment protocol (Kleinjung et al., 2005; Plewnia et al., 2007; Rossi et al., 2007; Smith et al., 2007)] it would be a bit too early to draw the conclusion that the small effect sizes might not possibly reflect clinically relevant changes especially taking into consideration the much higher effect sizes of the responder group. Even if this pilot study might have been designed with limited power presumptions, the results suggest at least that the applied combined study protocol did prove to be non-inferior in comparison to the established stimulation pattern of $1 \mathrm{~Hz}$ to temporal targets.

In this study all patients received rTMS over the left temporal cortex and in the combined group additionally over the right DLPFC. For both targets it remains a matter of debate, whether a more individualized strategy may not be more efficient. For the temporal cortex there are conflicting results whether stimulation ipsi- or contra-lateral to the perceived tinnitus laterality is more efficient (Frank et al., 2010; Khedr et al., 2010). We chose left temporal stimulation in all patients for better comparison with previous studies that investigated enhancement strategies for rTMS (Kleinjung et al., 2008, 2011; Langguth et al., 2008). With respect to frontal stimulation individualized targeting based on function imaging data revealed conflicting results as well (Kimbrell et al., 1999; Herwig et al., 2003) and more consistent antidepressant efficacy has been reported for low-frequency rTMS over the right DLPFC independent from imaging data (Schutter, 2010). 
There is growing evidence from many recent neuroimaging studies that the influence of non-auditory brain structures may have been underestimated in the pathophysiology of chronic tinnitus in the past. A study investigating long-range connectivity of brain areas in patients suffering from chronic tinnitus by means of magnetoencephalography detected mainly the prefrontal cortex and the orbitofrontal region as hubs in tinnitus related networks (Schlee et al., 2009b). Moreover with increasing tinnitus duration non-auditory areas seem to gain importance in tinnitus related networks in comparison to auditory areas (Schlee et al., 2009b). But not only the DLPFC and neighboring regions seem to be of decisive relevance; also the left hippocampus (Landgrebe et al., 2009), parahippocampus (Lockwood et al., 1998; Schecklmann et al., 2011), the anterior (Plewnia et al., 2007) and posterior cingulate cortex (Vanneste et al., 2010a; Schecklmann et al., 2011), the temporoparietal junction (=auditory association area; Shulman, 1995; Giraud et al., 1999; Lockwood et al., 1999; Gardner et al., 2002), the dorsolateral prefrontal cortex (Mirz et al., 2000; Voisin et al., 2006), and the cerebellum (Lanting et al., 2009) have consistently been shown to exert functional influence in chronic tinnitus.

Possibly other rTMS techniques with different target locations, frequencies, and stimulation protocols (e.g., burst protocols; Arfeller et al., 2009; Kleinjung and Langguth, 2009) might represent promising approaches. Very recently a new rTMS coil, the so-called double-cone-coil with increased stimulation depth in the brain, has been introduced. Based on the use of this device it has been shown that a direct modulating influence of rTMS can be

\section{REFERENCES}

Arfeller, C., Vonthein, R., Plontke, S. K., and Plewnia, C. (2009). Efficacy and safety of bilateral continuous theta burst stimulation (cTBS) for the treatment of chronic tinnitus: design of a three-armed randomized controlled trial. Trials 10, 74.

Beck, A. T., and Steer, R. A. (1984). Internal consistencies of the original and revised Beck Depression Inventory. J. Clin. Psychol. 40, 1365-1367.

Cheeran, B., Talelli, P., Mori, F., Koch, G., Suppa, A., Edwards, M., Houlden, H., Bhatia, K., Greenwood, R., and Rothwell, J. C. (2008). A common polymorphism in the brain-derived neurotrophic factor gene (BDNF) modulates human cortical plasticity and the response to rTMS. J. Physiol. (Lond.) 586, 5717-5725.

Cohen, J. (1988). Statistical Power for the Behavioral Sciences. Hillsdale, NJ: Erlbaum.

Crippa, A., Lanting, C. P., Van Dijk, P., and Roerdink, J. B. (2010). A diffusion tensor imaging study on the auditory system and tinnitus. Open. Neuroimag. J. 4, 16-25.

Davidson, R. J. (1992). Anterior cerebral asymmetry and the nature of emotion. Brain Cogn. 20, 125-151.
De Ridder, D., Elgoyhen, A. B., Romo, R., and Langguth, B. (2011). Phantom percepts: tinnitus and pain as persisting aversive memory networks. Proc. Natl. Acad. Sci. U.S.A. 108, 8075-8080.

Eichhammer, P., Langguth, B., Marienhagen, J., Kleinjung, T., and Hajak, G. (2003). Neuronavigated repetitive transcranial magnetic stimulation in patients with tinnitus: a short case series. Biol. Psychiatry 54, 862-865.

Frank, E., Schecklmann, M., Landgrebe, M., Burger, J., Kreuzer, P., Poeppl, T. B., Kleinjung, T., Hajak, G., and Langguth, B. (2011). Treatment of chronic tinnitus with repeated sessions of prefrontal transcranial direct current stimulation: outcomes from an openlabel pilot study. J. Neurol. doi: 10.1007/s00415-011-6189-4. [Epub ahead of print].

Frank, G., Kleinjung, T., Landgrebe, M., Vielsmeier, V., Steffenhagen, C., Burger, J., Frank, E., Vollberg, G., Hajak, G., and Langguth, B. (2010). Left temporal low-frequency rTMS for the treatment of tinnitus: clinical predictors of treatment outcome a retrospective study. Eur. J. Neurol. 17, 951-956. exerted to the limbic system, namely the anterior cingulate cortex (Hayward et al., 2007). In first clinical trials this new technique has been proven to be safe and its application is feasible and well tolerated (personal communication S Vanneste and D de Ridder).

Even if the present study has not been placebo controlled, the results further support the efficacy of low-frequency rTMS for the treatment of tinnitus as demonstrated in previous studies (Kleinjung et al., 2005; Plewnia et al., 2007; Rossi et al., 2007; Smith et al., 2007). The inter-individual variability has been high in both treatment groups, highlighting the relevance of a more individualized treatment approach. The limited accuracy of the coil positioning procedure over the DLPFC together with the large anatomic interindividual variability of the DLPFC in Brodman Area 9 and Brodman Area 46 (Herbsman et al., 2009) may play a role in this context as well as potential genetic influences on neuromodulatory effects as proposed for the BDNF polymorphisms (Cheeran et al., 2008). It has been shown that clinical characteristics have only limited value for predicting treatment outcome (Frank et al., 2010). Neuroimaging such as electro- or magnet-encephalography may be more promising for identifying patients who may respond well on specific stimulation protocols (Lorenz et al., 2010; Vanneste et al., 2011). This may lead to the development of individualized multisite-rTMS-stimulation techniques for the treatment of tinnitus, but also in other indications such as depression or chronic pain.

\section{ACKNOWLEDGMENTS}

We thank Helene Niebling and Sandra Pfluegl for their technical assistance in administering rTMS and collecting data.

Gardner, A., Pagani, M., Jacobsson, H., Lindberg, G., Larsson, S. A., Wagner, A., and Hallstrom, T. (2002). Differences in resting state regional cerebral blood flow assessed with 99mTc-HMPAO SPECT and brain atlas matching between depressed patients with and without tinnitus. Nucl. Med. Commun. 23, 429-439.

George, M. S., Wassermann, E. M., Williams, W. A., Callahan, A., Ketter, T. A., Basser, P., Hallett, M., and Post, R. M. (1995). Daily repetitive transcranial magnetic stimulation (rTMS) improves mood in depression. Neuroreport 6, 1853-1856.

Giraud, A. L., Chery-Croze, S., Fischer, G., Fischer, C., Vighetto, A., Gregoire, M. C., Lavenne, F., and Collet, L. (1999). A selective imaging of tinnitus. Neuroreport 10, 1-5.

Goebel, G., and Hiller, W. (1994). The tinnitus questionnaire. A standard instrument for grading the degree of tinnitus. Results of a multicenter study with the tinnitus questionnaire. HNO 42, 166-172.

Hallett, M. (2000). Transcranial magnetic stimulation and the human brain. Nature 406, 147-150.
Hayward, G., Mehta, M. A., Harmer, C., Spinks, T. J., Grasby, P. M., and Goodwin, G. M. (2007). Exploring the physiological effects of double-cone coil TMS over the medial frontal cortex on the anterior cingulate cortex: an $\mathrm{H} 2(15) \mathrm{O}$ PET study. Eur. J. Neurosci. 25, 2224-2233.

Herbsman, T., Avery, D., Ramsey, D., Holtzheimer, P., Wadjik, C., Hardaway, F., Haynor, D., George, M. S., and Nahas, Z. (2009). frontal coil location is associated with better repetitive transcranial magnetic stimulation antidepressant response. Biol. Psychiatry 66, 509-515.

Herwig, U., Abler, B., SchonfeldtLecuona, C., Wunderlich, A., Grothe, J., Spitzer, M., and Walter, $\mathrm{H}$. (2003). Verbal storage in a premotorparietal network: evidence from fMRI-guided magnetic stimulation. Neuroimage 20, 1032-1041.

Hesser, H., Weise, C., Rief, W., and Andersson, G. (2011). The effect of waiting: a meta-analysis of wait-list control groups in trials for tinnitus distress. J. Psychosom. Res. 70, 378-384. More lateral and anterior pre- 
Jastreboff, P. J. (1990). Phantom auditory perception (tinnitus): mechanisms of generation and perception. Neurosci. Res. 8, 221-254.

Khedr, E. M., Abo-Elfetoh, N., Rothwell, J. C., El-Atar, A., Sayed, E., and Khalifa, H. (2010). Contralateral versus ipsilateral rTMS of temporoparietal cortex for the treatment of chronic unilateral tinnitus: comparative study. Eur. J. Neurol. 17, 976-983.

Kimbrell, T. A., Little, J. T., Dunn, R. T., Frye, M. A., Greenberg, B. D., Wassermann, E. M., Repella, J. D., Danielson, A. L., Willis, M. W., Benson, B. E., Speer, A. M., Osuch, E., George, M. S., and Post, R. M. (1999). Frequency dependence of antidepressant response to left prefrontal repetitive transcranial magnetic stimulation (rTMS) as a function of baseline cerebral glucose metabolism. Biol. Psychiatry 46, 1603-1613.

Kleinjung, T., Eichhammer, P., Landgrebe, M., Sand, P., Hajak, G., Steffens, T., Strutz, J., and Langguth, B. (2008). Combined temporal and prefrontal transcranial magnetic stimulation for tinnitus treatment: a pilot study. Otolaryngol. Head Neck Surg. 138, 497-501.

Kleinjung, T., Eichhammer, P., Langguth, B., Jacob, P., Marienhagen, J., Hajak, G., Wolf, S. R., and Strutz, J. (2005). Long-term effects of repetitive transcranial magnetic stimulation (rTMS) in patients with chronic tinnitus. Otolaryngol. Head Neck Surg. 132, 566-569.

Kleinjung, T., Fischer, B., Langguth, B., Sand, P., Hajak, G., Dvorakova, J., and Eichhammer, P. (2007a). Validation of the German-version Tinnitus Handicap Inventory (THI). Psychiatr. Prax. 34, 140-142.

Kleinjung, T., Steffens, T., Sand, P., Murthum, T., Hajak, G., Strutz, J., Langguth, B., and Eichhammer, P. (2007b). Which tinnitus patients benefit from transcranial magnetic stimulation? Otolaryngol. Head Neck Surg. 137, 589-595.

Kleinjung, T., and Langguth, B. (2009). Strategies for enhancement of transcranial magnetic stimulation effects in tinnitus patients. Int. Tinnitus J. $15,154-160$.

Kleinjung, T., Steffens, T., Landgrebe, M., Vielsmeier, V., Frank, E., Burger, J., Strutz, J., Hajak, G., and Langguth, B. (2011). Repetitive transcranial magnetic stimulation for tinnitus treatment: no enhancement by the dopamine and noradrenaline reuptake inhibitor bupropion. Brain Stimul. 4, 65-70.
Landgrebe, M., Langguth, B., Rosengarth, K., Braun, S., Koch, A., Kleinjung, T., May, A., De Ridder, D., and Hajak, G. (2009). Structural brain changes in tinnitus: grey matter decrease in auditory and nonauditory brain areas. Neuroimage 46 , 213-218.

Landgrebe, M., Zeman, F., Koller, M., Eberl, Y., Mohr, M., Reiter, J., Staudinger, S., Hajak, G., and Langguth, B. (2010). The Tinnitus Research Initiative (TRI) database: a new approach for delineation of tinnitus subtypes and generation of predictors for treatment outcome. BMC Med. Inform. Decis. Mak. 10, 42. doi: 10.1186/1472-6947-10-42

Langguth, B., Kleinjung, T., Frank, E., Landgrebe, M., Sand, P., Dvorakova, J., Frick, U., Eichhammer, P., and Hajak, G. (2008). High-frequency priming stimulation does not enhance the effect of low-frequency rTMS in the treatment of tinnitus. Exp. Brain Res. 184, 587-591.

Langguth, B., Landgrebe, M., Kleinjung, T., Sand, G. P., and Hajak, G. (2011) Tinnitus and depression. World J. Biol. Psychiatry 12, 489-500.

Lanting, C. P., De Kleine, E., Eppinga, R. N., and Van Dijk, P. (2010). Neural correlates of human somatosensory integration in tinnitus. Hear. Res. 267, 78-88.

Lanting, C. P., De Kleine, E., and Van Dijk, P. (2009). Neural activity underlying tinnitus generation: results from PET and fMRI. Hear. Res. 255, 1-13.

Leaver, A. M., Renier, L., Chevillet, M. A., Morgan, S., Kim, H. J., and Rauschecker, J. P. (2011). Dysregulation of limbic and auditory networks in tinnitus. Neuron 69, 33-43.

Lockwood, A. H., Salvi, R. J., Burkard, R. F., Galantowicz, P. J., Coad, M. L., and Wack, D. S. (1999). Neuroanatomy of tinnitus. Scand. Audiol. Suppl. 51, 47-52.

Lockwood, A. H., Salvi, R. J., Coad, M. L., Towsley, M. L., Wack, D. S., and Murphy, B. W. (1998). The functional neuroanatomy of tinnitus: evidence for limbic system links and neural plasticity. Neurology 50, 114-120.

Lorenz, I., Muller, N., Schlee, W., Langguth, B., and Weisz, N. (2010). Short-term effects of single repetitive TMS sessions on auditory evoked activity in patients with chronic tinnitus. J. Neurophysiol. 104, 1497-1505.

May, A., Hajak, G., Ganssbauer, S., Steffens, T., Langguth, B., Kleinjung, T., and Eichhammer, P. (2007). Structural brain alterations follow- ing 5 days of intervention: dynamic aspects of neuroplasticity. Cereb. Cortex 17, 205-210.

Mirz, F., Gjedde, A., Ishizu, K., and Pedersen, C. B. (2000). Cortical networks subserving the perception of tinnitus - a PET study. Acta Otolaryngol. Suppl. 543, 241-243.

Moller, A. R. (2003). Pathophysiology of tinnitus. Otolaryngol. Clin. North Am. 36, 249-266, v-vi.

Murphy, B., Herrman, H., Hawthorne, G., Pinzone, T., and Evert, $\mathrm{H}$. (2000). Australian WHOQoL Instruments: User's Manual and Interpretation Guide. Melbourne: Australian WHOQol Field Study Centre.

Norena, A., Cransac, H., and CheryCroze, S. (1999). Towards an objectification by classification of tinnitus. Clin. Neurophysiol. 110 666-675.

Paus, T., Castro-Alamancos, M. A., and Petrides, M. (2001). Corticocortical connectivity of the human mid-dorsolateral frontal cortex and its modulation by repetitive transcranial magnetic stimulation. Eur. J. Neurosci. 14, 1405-1411.

Plewnia, C., Reimold, M., Najib, A. Reischl, G., Plontke, S. K., and Gerloff, C. (2007). Moderate therapeutic efficacy of positron emission tomography-navigated repetitive transcranial magnetic stimulation for chronic tinnitus: a randomised, controlled pilot study. $J$. Neurol. Neurosurg. Psychiatr. 78 152-156.

Rauschecker, J. P., Leaver, A. M., and Muhlau, M. (2010). Tuning out the noise: limbic-auditory interactions in tinnitus. Neuron 66, 819-826.

Ridding, M. C., and Rothwell, J. C. (2007). Is there a future for therapeutic use of transcranial magnetic stimulation? Nat. Rev. Neurosci. 8, 559-567.

Rossi, S., De Capua, A., Ulivelli, M., Bartalini, S., Falzarano, V., Filippone, G., and Passero, S. (2007). Effects of repetitive transcranial magnetic stimulation on chronic tinnitus: a randomised, crossover, double blind, placebo controlled study. J. Neurol. Neurosurg. Psychiatr. 78, 857-863.

Schecklmann, M., Landgrebe, M., Poeppl, T., Kreuzer, P., Maenner, P. Marienhagen, J., Wack, D., Kleinjung, T., Hajak, G., and Langguth, B. (2011). Neuronal correlates of tinnitus duration and distress: a positron emission tomography study. Hum. Brain Mapp. doi 10.1002/hbm.21426. [Epub ahead of print].

Schlee, W., Hartmann, T., Langguth, B., and Weisz, N. (2009a). Abnor- mal resting-state cortical coupling in chronic tinnitus. BMC Neurosci. 10, 11. doi: 10.1186/1471-2202-10-11

Schlee, W., Mueller, N., Hartmann, T., Keil, J., Lorenz, I., and Weisz, N. (2009b). Mapping cortical hubs in tinnitus. BMC Biol. 7, 80. doi: 10.1186/1741-7007-7-80

Schlee, W., Weisz, N., Bertrand, O., Hartmann, T., and Elbert, T. (2008). Using auditory steady state responses to outline the functional connectivity in the tinnitus brain. PLoS ONE 3, e3720. doi: 10.1371/journal.pone.0003720

Schmidt, B., and Hanslmayr, S. (2009). Resting frontal EEG alpha-asymmetry predicts the evaluation of affective musical stimuli. Neurosci. Lett. 460, 237-240.

Schutter, D. J. (2010). Quantitative review of the efficacy of slowfrequency magnetic brain stimulation in major depressive disorder. Psychol. Med. 40, 1789-1795.

Shulman, A. (1995). A final common pathway for tinnitus - the medial temporal lobe system. Int. Tinnitus J. 1,115-126.

Siebner, H. R., Filipovic, S. R., Rowe, J. B., Cordivari, C., Gerschlager, W., Rothwell, J. C., Frackowiak, R. S., and Bhatia, K. P. (2003). Patients with focal arm dystonia have increased sensitivity to slowfrequency repetitive TMS of the dorsal premotor cortex. Brain 126, 2710-2725.

Siebner, H. R., Lang, N., Rizzo, V., Nitsche, M. A., Paulus, W., Lemon, R. N., and Rothwell, J. C. (2004). Preconditioning of lowfrequency repetitive transcranial magnetic stimulation with transcranial direct current stimulation: evidence for homeostatic plasticity in the human motor cortex. J. Neurosci. 24, 3379-3385.

Siebner, H. R., Peller, M., Willoch, F., Minoshima, S., Boecker, H., Auer, C., Drzezga, A., Conrad, B. and Bartenstein, P. (2000). Lasting cortical activation after repetitive TMS of the motor cortex: a glucose metabolic study. Neurology 54, 956-963.

Smith, J. A., Mennemeier, M., Bartel, T., Chelette, K. C., Kimbrell, T., Triggs, W., and Dornhoffer, J. L. (2007). Repetitive transcranial magnetic stimulation for tinnitus: a pilot study. Laryngoscope 117, 529-534.

Vanneste, S., and De Ridder, D. (2011). Bifrontal transcranial direct current stimulation modulates tinnitus intensity and tinnitusdistress-related brain activity. Eur. J. Neurosci. 34, 605-614. 
Vanneste, S., Focquaert, F., Van De Heyning, P., and De Ridder, D. (2011). Different resting state brain activity and functional connectivity in patients who respond and not respond to bifrontal tDCS for tinnitus suppression. Exp. Brain Res. 210, 217-227.

Vanneste, S., Plazier, M., Der Loo, E., De Heyning, P. V., Congedo, M., and De Ridder, D. (2010a). The neural correlates of tinnitusrelated distress. Neuroimage 52, 470-480.

Vanneste, S., Plazier, M., Ost, J., Van Der Loo, E., Van De Heyning, P., and De Ridder, D. (2010b). Bilateral dorsolateral prefrontal cortex modulation for tinnitus by transcranial direct current stimulation: a preliminary clinical study. Exp. Brain Res. 202, 779-785.

Voisin, J., Bidet-Caulet, A., Bertrand, O., and Fonlupt, P. (2006). Listening in silence activates auditory areas: a functional magnetic resonance imaging study. J. Neurosci. 26, 273-278.

Weisz, N., Dohrmann, K., and Elbert, T. (2007a). The relevance of spontaneous activity for the coding of the tinnitus sensation. Prog. Brain Res. 166, 61-70.

Weisz, N., Muller, S., Schlee, W., Dohrmann, K., Hartmann, T., and Elbert, T. (2007b). The neural code of auditory phantom perception. J. Neurosci. 27, 1479-1484.

Conflict of Interest Statement: The authors declare that the research was conducted in the absence of any commercial or financial relationships that could be construed as a potential conflict of interest.

Received: 12 August 2011; accepted: 12 October 2011; published online: 04 November 2011.

Citation: Kreuzer PM, Landgrebe $M$, Schecklmann M, Poeppl TB, Vielsmeier
$V$, Hajak G, Kleinjung T and Langguth $B$ (2011) Can temporal repetitive transcranial magnetic stimulation be enhanced by targeting affective components of tinnitus with frontal rTMS? A randomized controlled pilot trial. Front. Syst. Neurosci. 5:88. doi: 10.3389/fnsys.2011.00088

Copyright (c) 2011 Kreuzer, Landgrebe, Schecklmann, Poeppl, Vielsmeier, Hajak, Kleinjung and Langguth. This is an openaccess article subject to a non-exclusive license between the authors and Frontiers Media SA, which permits use, distribution and reproduction in other forums, provided the original authors and source are credited and other Frontiers conditions are complied with. 